\title{
Einsatz virtueller Transportsimulatoren zum umweltbewußten Gefahrensituationstraining
}

\author{
Kirsten Wechsel, Studentin
}

\section{Begriffsdefinition}

Simulation ist die modellhafte Darstellung oder Nachbildung bestimmter Aspekte eines vorhandenen oder zu entwickelnden Systems, insbesondere auch eines Zeitverhaltens. Die Simulation erlaubt Untersuchungen oder Manipulationen, deren Durchführung am eigentlichen System zu gefährlich, zu teuer oder unmöglich ist. Ein Simulator ist dementsprechend ein Gerät oder System, mit dem gewisse Verhaltensweisen und Eigenschaften eines Systems nachgeahmt werden können.

\section{Ausgangssituation}

Der weltweite Handel mit gefährlichen Gütern hat einen großen Umfang erreicht und nimmt ständig zu. Allein in der Bundesrepublik Deutschland werden jährlich über 400 Millionen Tonnen Gefahrgüter befördert. Nachfolgende Grafik veranschaulicht die Verteilung auf die einzelnen Verkehrsträger.

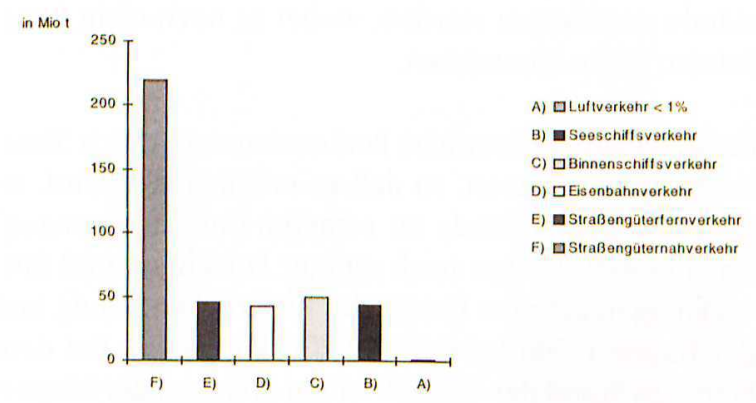

Quelle: Statistisches Bundesant 1995

Die Grafik zeigt deutlich, daß ein großer Teil der gefährlichen Güter auf der Straße befördert wird. Trotz der großen Anzahl von Sicherheitsbestimmungen kommt es aber immer wieder zu Unfällen, die gerade beim Transport von gefährlichen Gütern enorme Folgen haben können.

In der nachstehenden Grafik werden die Ursachen von Unfällen bei Gefahrguttransporten veranschaulicht.
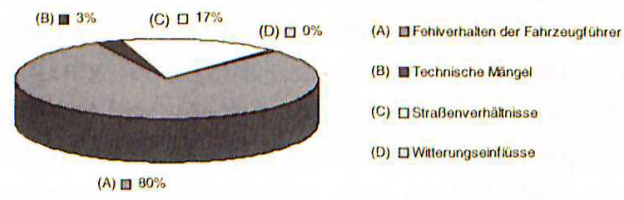

Quelle: Statistisches Bundesamt 1995

Die Auswertung der Statistiken des Statistischen Bundesamtes ergab, daß der größte Teil der Unfälle bei Gefahrguttransporten auf das Fehlverhalten der Fahr- zeugführer zurüickzufuihren ist. Eine weitaus kleinere Zahl von Unfällen ist auf technische Mängel, Straßenverhältnisse oder Witterungseinflüsse zurückzuführen. Die Statistiken beweisen, daß $80 \%$ aller Unfälle durch richtiges Verhalten der Fahrzeugfuihrer vermieden werden könnte. Das richtige Verhalten muß aber trainiert werden, da gerade im Gefahrguttransport die Folgen eines Unfalls fuir den Fahrer, unbeteiligte Menschen und die Umwelt meist nicht absehbar sind.

\section{Innovative Zielstellung}

Seit Jahren werden zur Ausbildung von Betriebspersonal, für komplexe Anlagen wie z.B. Kraftwerke, Flugzeuge, Schiffe, Panzer und Raumfähren, Simulatoren eingesetzt. Mit Hilfe dieser Simulatoren werden u. a. Flugzeugfuihrer und Schiffskapitäne mit der Bedienung und den spezifischen Eigenschaften ihrer Fahrzeuge vertraut gemacht, noch bevor sie bei einem wirklichen Einsatz eventuell gefährlichen Situationen ausgesetzt sind. Den Erfolg eines solchen Trainings beweisen nicht zuletzt die Mondlandungen oder die Space-Shuttle-Fliige, denn ihre Besatzungen konnten nur mit Simulatoren trainieren. Ein Aspekt, der fuir den Einsatz von Fahrsimulatoren bei der Aus- und Weiterbildung von Fahrschülern spricht, ist die Unabhängigkeit von den realen Straßensituationen, wie z. B. Wetter, Landschaft, Verkehrsaufkommen und Tageszeiten. Weiterhin kann ein Fahrsimulator prinzipielle Schwächen des Fahrenlernens auf der Straße uiberwinden. Jede Verkehrssituation, in die Fahrschüler kommen, ist einzigartig, sie läßt sich nicht wiederholen. Fahrsituationen wechseln rasch und ständig und sie lassen sich nur begrenzt arrangieren. Es ist oft dem Zufall überlassen, ob sich gerade dann, wenn der Fahrschüler z.B. das Überholen lernen soll, genügend Situationen bieten, in denen er dies tatsächlich lernen kann, und schließlich kommen bestimmte Fahrsituationen gar nicht vor.

Der große Vorteil des Fahrsimulators ist, daß seine Software Verkehrssituationen gezielt auswählen, wiederholen und in Szenenfolgen anordnen kann, die das Lernen optimieren können.

Zur Zeit gibt es die ersten Fahrsimulatoren, die so aufgebaut sind, daß sich der Fahrer in einer echten, sich bewegenden Fahrerkabine befindet. Eine solche Fahrerkabine ist mit allen notwendigen Armaturen und Bedienelementen eines bestimmten Fahrzeugmodells ausgestattet und wird vor einer halbkreisförmigen Panoramafläche positioniert. Auf diese Fläche werden dann die aus der Fahrerkabine sichtbare Umgebung projektiert. Da diese Kabinen aus technischen Gründen einen sehr großen Platzbedarf haben, werden derartige Simulato- 
ren in großen, zumeist eigens dafür eingerichtete Trainingszentren, aufgebaut. Aufgrund technischer Gegebenheiten kann man mit diesen Systemen ausschließlich die Fahrzeugfuihrung simulieren. Es ist nicht möglich vor- und nachgelagerten Aktivitäten wie Be- und Entladen nachzuempfinden. Da neben dem hohen Platzbedarf auch enorme Kosten auf den Betreiber eines solchen System zukommen, sind solche Simulatoren nicht für eine flächendeckende Ausrüstung der Fahrschulen und Betriebe geeignet.

Ziel ist es nun, eine Low-Cost-Version eines Simulators zu entwickeln, der unabhängig von großen Trainingszentren zum Einsatz gebracht werden kann. Der Simulator soll mit seinen Hard- und Softwarekomponenten so gestaltet sein, daß es auch kleinen Trainingszentren, Fahrschulen oder Betrieben möglich ist, ihn einzusetzen. Das System sollte dabei folgende Voraussetzungen erfiillen:

- geringer Platzbedarf und kleines Gewicht

- transportabel und geringer technischer Aufwand (Hydraulik, Pneumatik)

- unabhängig von Fahrzeugtypen

Der Simulator soll in der Lage sein, sowohl die Fahrt selbst als auch die vor- und nachgelagerten Aktivitäten zu simulieren. Solche Aktivitäten könnten z.B. sein: Beund Entladen, Überprüfen der Verkehrssicherheit und Fahrtvorbereitungen. Neben dem Einsatz zum Trainieren gefährlicher Situationen, kann auch die Fahrweise selbst geschult werden (z.B. zur Verringerung des Kraftstoffverbrauchs oder die Verbesserung der Selbsteinschätzung des Fahrers).

\section{Vorstellungen zur technischen Umsetzung}

Die Projektion erfolgt iiber ein HMD (Head Mounted Display). Ein HMD ermöglicht ein dreidimensionales Sehen mit einer Bewegungsfreiheit von $360^{\circ}$ sowie die Echtzeitsensorik für alle Kopfbewegungen. Über das HMD können die Geräusche eingespielt werden. Die Bewegungen könnten mit Hilfe einer hydraulischen Bewegungsplattform simuliert werden. Eine solche Plattform ist in allen Freiheitsgraden beweglich. Die auftretenden translatorischen Beschleunigungen sowie die Rotationsgeschwindigkeiten werden entsprechend der visuellen Darstellung an die Bewegungsplattform weitergegeben.

Damit interaktiv in den Ablauf der Simulation eingegriffen werden kann, stehen dem „Schüler“ die verschiedenen Eingabegeräte wie Datenhandschuhe bzw. Datenanzug, Bedienelemente (wie z.B. Lenkrad, Pedale) und Armaturen (z.B. für Geschwindigkeitsanzeige, Drehzahlmesser) zur Verfuigung. Die Bedienelemente, Armaturen sowie der Fahrersitz befinden sich auf der Bewegungsplattform. Auf das HMD, in Höhe der Augen, wird eine kleine Kamera angebracht, welche es dem „Schüler“ erlaubt, die Bedienelemente, Armaturen sowie seine Arme und Beine zu sehen. Die Bedienelemente, Armaturen sowie der Fahrersitz und somit auch der „Schiiler" sind hierbei von blauen Leinwänden umgeben, die es ermöglichen, die virtuellen Bilder mit den realen Bildern der Kamera zu verbinden. Dieses Verfahren wird bereits in den virtuellen Fernsehstudios praktiziert.

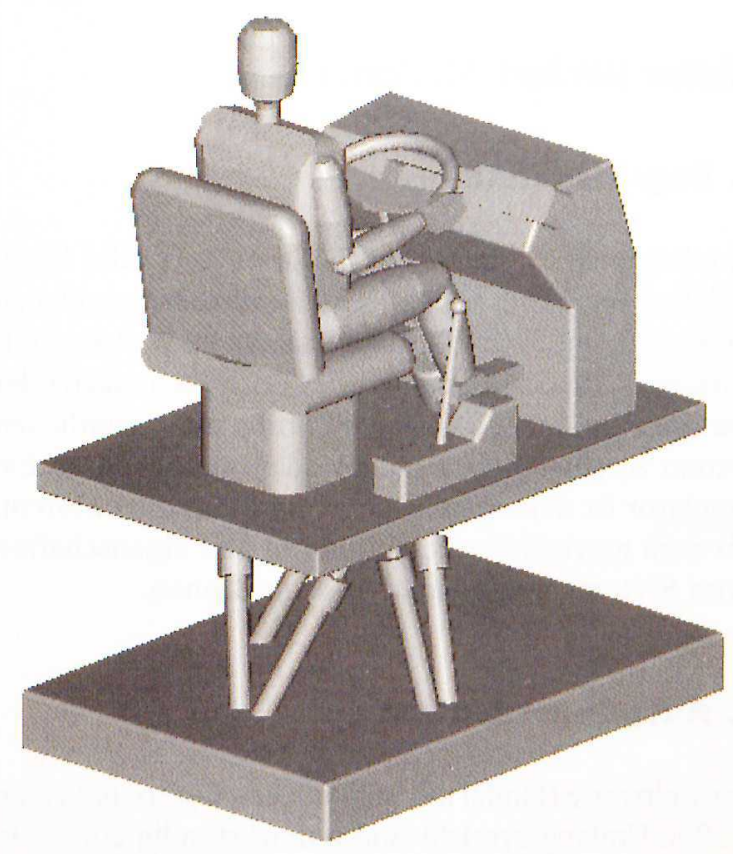

Bild 1: Vorstellungen zur technischen Umsetzung

Mit Hilfe des Datenhandschuhs können virtuelle Gegenstände verschoben werden, wobei es noch nicht möglich ist, diese zu ertasten.

Es ist geplant, körperliche Bedienelemente durch Simulationen zu ersetzen, so daß es möglich sein wird, simulierte Gegenstände zu ertasten und zu bewegen. Dazu bedarf es aber noch einiger Forschung und Entwicklungen auf dem Gebiet der Kraftrüickkopplung und der Haptik ( Taktiles Gefühl / Rüickkopplung). Bei dem heutigen Stand der Technik ist ein Ersetzen der körperlichen Bedienelemente durch Simulationen noch nicht möglich.

\section{Visuelle Simulation}

Der Entwicklungsstand der Visualisierung macht es möglich, virtuelle Welten zu erschaffen. Um die Landschaft so realitätsnah wie möglich zu gestalten, wäre es von großem Nutzen, in die Programmierung der Module Videoaufnahmen einzubeziehen. Durch die vielen Kombinationsmöglichkeiten der Umgebung, Straße, Situationen und Wetter kann die Fahrstrecke beliebig verlängert werden. Es sollte aber sichergestellt werden, daß die Jahreszeiten und Witterungseinflüsse im Laufe der Route beibehalten bzw. aufeinander abgestimmt werden. Weiterhin muß berïcksichtigt werden, daß innerhalb des Straßen und Landschaftsbildes gewisse Abhängigkeiten berücksichtigt werden. Das Bildmaterial ist so zu entwickeln, daß es durch entsprechende Computerprogramme zusammengesetzt werden kann. Es werden 
einzelne Module programmiert, die dann durch einen Zufallsgenerator beliebig zusammengesetzt werden können.

Derartige Module können sein:

- Umgebung (Häuser, Läden/Einkaufszentren, Tankstellen, Schule, Stadion, Parkhaus, Museum, Bahnhof)

- Landschaft (Hiigel, Bäume, Weide, Wiese, See, Bauernhof)

- Straßenbild (Straße: geradeaus, Kurve rechts, Kurve links, Doppelkurve, Steigung, Gefälle, Abzweig rechts, Abzweig links, Kreuzung mit und ohne Ampel)

- Andere Verkehrsteilnehmer (Pkw/Motorräder/Lkw, Busse, Radfahrer, Fußgänger)

- Gefahrensituationen (Wildwechsel, Reifen platzt, Bremsen versagen)

- Wettereinfluisse (nasse Fahrbahn, glatte Fahrbahn, schlechte Sicht durch Regen, Schnee, oder Nebel, Tag, Nacht)

\section{Simulation der Bewegung}

Zu einer realitätsnahen Simulation gehört es auch, die Bewegungen zu simulieren. Um die Bewegungen, die in einem Fahrzeug entstehen, simulieren zu können, muß die Fahrphysik beachtet werden, da diese sich nicht verändern läßt. Der Kraftfahrer muß sich nach ihr richten.

Bei den verschiedenen Fahrmanövern treten unterschiedliche Kräfte auf, die auf den Fahrer uibertragen werden müssen. Unterschiedliche Fahrmanöver sind: Anfahren, Beschleunigen, Bremsen, Lenken, Anhalten, Kurvenfahrten, Bergfahrten und Talfahrten. Weiterhin wirken verschiedene Kräfte, wenn sich die Witterungssituation verändert. Derartige veränderte Witterungsbedingungen können Wind, Nässe, Eis oder Schnee sein. Bei dem Fahrzeugverhalten spielt neben den Fahrmanövern und der Witterung auch die Ladung und der Fahrzeugzustand bzw. die Fahrbahnart und dessen Zustand sowie das Verhalten des Fahrzeugfuihrers und die vorhandenen technischen Möglichkeiten wie z.B. Motorbremse, Automatische Blockierverhinderer oder Bremskraftregler eine wesentliche Rolle.

\section{Ausblick}

Weiterfiihrend ist geplant, den Simulator in der Technischen Fachhochschule Wildau in die Realität umzusetzen. Für die Weiterentwicklung und die benötigten Geräte wurde bereits ein Antrag auf Förderung von anwendungsorientierter Forschung gestellt. Während der Arbeiten an diesem Projekt fanden sich bereits interessierte Fahrschulen und Speditionen und boten ihre Kooperation an. Sollte Ihrerseits noch Interesse an einer Mitarbeit bei diesem Projekt bestehen, so können Sie sich gern an uns wenden. Interessenten melden sich bei Prof. Hentschel unter der Telefonnummer (03375) 508 927 oder per Fax (03375) 508928.

\section{Verfasser}

Kirsten Wechsel, Studentische Hilfskraft Technische Fachhochschule Wildau Fachbereich Ingenieurwesen/Wirtschaftsingenieurwesen Fachgebiet Produktionslogistik

Tel.: (03375) 508924 